\title{
Parity-doublet representation of Majorana fermions and neutron oscillation
}

\author{
Fujikawa, Kazuo
}

2016-12-02

Fujikawa , K \& Tureanu , A 2016 , ' Parity-doublet representation of Majorana fermions and neutron oscillation ' , Physical Review D , vol. 94 , no. 11 , 115009 . https://doi.org/10.1103/PhysRevD.94.115009

http://hdl.handle.net/10138/183456

https://doi.org/10.1103/PhysRevD.94.115009

cc_by

publishedVersion

Downloaded from Helda, University of Helsinki institutional repository.

This is an electronic reprint of the original article.

This reprint may differ from the original in pagination and typographic detail.

Please cite the original version. 


\title{
Parity-doublet representation of Majorana fermions and neutron oscillation
}

\author{
Kazuo Fujikawa ${ }^{1,2}$ and Anca Tureanu ${ }^{1}$ \\ ${ }^{1}$ Department of Physics, University of Helsinki, P.O. Box 64, FIN-00014 Helsinki, Finland \\ ${ }^{2}$ Quantum Hadron Physics Laboratory, RIKEN Nishina Center, Wako 351-0198, Japan
}

(Received 11 September 2016; published 2 December 2016)

\begin{abstract}
We present a parity-doublet theorem for the representation of the intrinsic parity of Majorana fermions, which is expected to be useful also in condensed matter physics, and it is illustrated to provide a criterion of neutron-antineutron oscillation in a Bardeen-Cooper-Schrieffer type of effective theory with $\Delta B=2$ baryon number-violating terms. The $C P$ violation in the present effective theory causes no direct $C P$-violating effects in the oscillation itself, which is demonstrated by the exact solution, although it influences the neutron electric dipole moment in the leading order of small $\Delta B=2$ parameters. An analog of Bogoliubov transformation, which preserves $P$ and $C P$, is crucial in the analysis.
\end{abstract}

DOI: 10.1103/PhysRevD.94.115009

\section{INTRODUCTION}

The Majorana fermions have received much attention recently not only in particle physics [1] but also in condensed matter physics [2]. The Majorana fermions are defined by the condition $\psi(x)=C \bar{\psi}^{T}(x)=\psi^{c}(x)$, where $C=i \gamma^{2} \gamma^{0}$ stands for the charge conjugation matrix [3].

We start with a neutral Dirac fermion $n(x)$ and define the combinations $\psi_{ \pm}(x)=\frac{1}{\sqrt{2}}\left[n(x) \pm n^{c}(x)\right]$, which satisfy $\psi_{ \pm}^{c}(x)= \pm \psi_{ \pm}(x)$, showing that $\psi_{+}(x)$ and $\psi_{-}(x)$ are Majorana fields. We treat the fermion with $\psi_{-}^{c}(x)=$ $-\psi_{-}(x)$ also as a Majorana fermion. It is well known $[3,4]$ that, in theories in which the fermion number is conserved, the discrete symmetry such as parity can generally be defined with an arbitrary phase freedom $\delta$, $n(x) \rightarrow e^{i \delta} \gamma^{0} n(t,-\vec{x})$. However, if fermion number is not conserved, the equivalence of parity operators under a phase transformation fails. As working definitions of $C, P$, and $T$ even in a theory with fermion number violation, we use the conventional definitions given in Ref. [3]. One can then confirm that the parity operation with vanishing phase $\delta=0$, which is the most common definition $[3,4]$ and called " $\gamma^{0}$ parity" in the following when we specify it more precisely, namely,

$$
n(x) \rightarrow \gamma^{0} n(t,-\vec{x}), \quad n^{c}(x) \rightarrow-\gamma^{0} n^{c}(t,-\vec{x}),
$$

which satisfy $\mathrm{P}^{2}=1$, leads to a doublet representation $\left\{\psi_{+}(x), \psi_{-}(x)\right\}$,

$$
\psi_{ \pm}(x) \rightarrow \gamma^{0} \psi_{\mp}(t,-\vec{x}) .
$$

Only when the two fermions $\psi_{ \pm}(x)$ are degenerate is this doublet representation consistent with dynamics [4]. The mass splitting in $\psi_{ \pm}(x)$ inevitably breaks the $\gamma^{0}$ parity as a symmetry of the Lagrangian.
On the other hand, one confirms that parity for an isolated single Majorana fermion is consistently defined only by " $i \gamma^{0}$ parity" with $\delta=\pi / 2$,

$$
n(x) \rightarrow i \gamma^{0} n(t,-\vec{x}), \quad n^{c}(x) \rightarrow i \gamma^{0} n^{c}(t,-\vec{x}),
$$

namely, by (see Ref. [4]),

$$
\psi_{ \pm}(x) \rightarrow i \gamma^{0} \psi_{ \pm}(t,-\vec{x}),
$$

which is consistent with the reality of $\psi_{ \pm}(x)$ in the Majorana representation in which $\gamma^{0}$ is Hermitian but purely imaginary. The phase freedom $\delta$ is thus fixed by the Majorana condition and $\mathrm{P}^{2}=-1$. This transformation rule (4) by itself does not tell the presence or absence of the mass splitting of $\psi_{ \pm}(x)$.

The intrinsic parity of Majorana fermions, in particular, the parity-doublet theorem stated below, are shown to play main roles in the discussion of neutron-antineutron oscillation [5,6], which has received attention recently [7-10].

\section{EFFECTIVE $\Delta B=2$ LAGRANGIAN FOR THE NEUTRON}

In the analysis of possible baryon-number violation and neutron oscillation, one can study essential aspects by analyzing the quadratic effective Hermitian Lagrangian with general $\Delta B=2$ terms added,

$$
\begin{aligned}
\mathcal{L}= & \bar{n}(x) i \gamma^{\mu} \partial_{\mu} n(x)-m \bar{n}(x) n(x) \\
& -\frac{i}{2} \epsilon_{1}\left[e^{i \alpha} n^{T}(x) C n(x)-e^{-i \alpha} \bar{n}(x) C \bar{n}^{T}(x)\right] \\
& -\frac{i}{2} \epsilon_{5}\left[n^{T}(x) C \gamma_{5} n(x)+\bar{n}(x) C \gamma_{5} \bar{n}^{T}(x)\right],
\end{aligned}
$$

where $m, \epsilon_{1}, \epsilon_{5}$, and $\alpha$ are real parameters. The most general quadratic Hermitian Lagrangian is written in the form (5) using the phase freedom of $n(x) \rightarrow n(x)=e^{i \beta} n^{\prime}(x)$; under 
this change of naming the field, the physical quantities in (5) such as mass eigenvalues are obviously invariant. But once one defines $n^{c}(x) \equiv C \bar{n}^{T}$, we have $n^{c}(x)=e^{-i \beta} n^{\prime c}(x)$, and thus the $C$ and $C P$ properties of the solutions of the Lagrangian (5) are changed. The first $\Delta B=2$ term with real $\epsilon_{1}$ breaks the $\gamma^{0}$ parity, while the second $\Delta B=2$ term with real $\epsilon_{5}$ preserves the $\gamma^{0}$ parity. The term with $\epsilon_{5}$ written in the variables in (5) preserves $C$ and $C P$. (In contrast, the first $\Delta B=2$ term preserves $i \gamma^{0}$ parity, while the second $\Delta B=2$ term breaks $i \gamma^{0}$ parity.) An analogy of neutron oscillation in (5) to Bardeen-Cooper-Schrieffer (BCS) theory was emphasized at the early stage of the study of neutron oscillation [11].

We assume that the baryon number of the neutron in our effective theory is fixed by strong interactions, and thus a parity-violating $\Delta B=0$ mass term $-\delta m^{\prime} \bar{n} i \gamma_{5} n$ induced by the QCD $\theta$-vacuum, for example, should be added to a possible parity-violating mass term induced by $\Delta B=2$ interactions discussed later.

The model (5) with $\alpha=0$, which preserves $C P$, is related to the single-flavor neutrino model in Ref. [1] if one replaces $n(x)$ by $\nu(x)$ and suitably adjusts the phase of the neutrino in the latter model. The mass eigenvalues are given by

$$
M_{ \pm}=\sqrt{m^{2}+\epsilon_{5}^{2}} \pm \epsilon_{1} .
$$

For the purpose of the analysis of $C P$ violation later, the Hermitian Lagrangian (5) with $\alpha=-\pi / 2$ is interesting. For this choice, the second term with $\epsilon_{1}$ breaks $\gamma^{0}$ parity and $C P$, although $\mathrm{C}$ is a good symmetry of the total Lagrangian. The Lagrangian with $\alpha=-\pi / 2$ is exactly solved in terms of the solutions of

$$
\left(i \not \partial-M_{ \pm}\right) \psi_{ \pm}(x)=0,
$$

with mass eigenvalues

$$
M_{ \pm}=\sqrt{\left(m \pm \epsilon_{1}\right)^{2}+\epsilon_{5}^{2}} .
$$

The neutron fields are then given by

$$
\frac{1}{\sqrt{2}}\left(\begin{array}{c}
n(x)+n^{c}(x) \\
n(x)-n^{c}(x)
\end{array}\right)=e^{-i \Theta \gamma_{5}} e^{-i \tau_{3} \gamma_{5} \bar{\Theta}}\left(\begin{array}{l}
\psi_{+}(x) \\
\psi_{-}(x)
\end{array}\right),
$$

where the chiral phase factors are defined by

$$
M_{ \pm} e^{2 i \tilde{\theta}_{ \pm} \gamma_{5}}=m \pm \epsilon_{1} \pm i \epsilon_{5} \gamma_{5},
$$

and $\Theta \equiv \frac{1}{2}\left(\tilde{\theta}_{+}+\tilde{\theta}_{-}\right)$and $\bar{\Theta} \equiv \frac{1}{2}\left(\tilde{\theta}_{+}-\tilde{\theta}_{-}\right)$. The charge conjugation properties of $\psi_{ \pm}$are [12]

$$
\psi_{+}^{c}(x)=\psi_{+}(x), \quad \psi_{-}^{c}(x)=-\psi_{-}(x)
$$

and are thus Majorana fermions, which are consistent with the $C$ transformation of $n$ and $n^{c}$ in (9) and also consistent with the $C$ invariance of (5) (with $\alpha=-\pi / 2$ ). One can confirm that the doublet representation of parity

$$
\psi_{ \pm}(x) \rightarrow \gamma^{0} \psi_{\mp}(t,-\vec{x})
$$

does not induce the $\gamma^{0}$-parity transformation of $n$ and $n^{c}$ for $\Theta \neq 0$ in (9), reflecting the $P$ (and thus $C P$ ) violation for $\epsilon_{1} \epsilon_{5} \neq 0$ (for $\epsilon_{1} \epsilon_{5}=0, \Theta=0$ ) in addition to the dynamical $P$ breaking $M_{+} \neq M_{-}$for $\epsilon_{1} \neq 0$ in (5).

It is confirmed that the $C P$ violation in the Lagrangian (5) (with $\alpha \neq 0$ ) is not eliminated by any phase choice of the neutron field $n(x) \rightarrow e^{i \beta} n(x)$ for real $\epsilon_{1} \neq 0$ and $\epsilon_{5} \neq 0$, and in this sense, it is intrinsic; $\epsilon_{1} \epsilon_{5} \neq 0$ is a necessary condition of $C P$ violation, and parity is inevitably broken.

\section{PARITY-DOUBLET THEOREM}

The presence of the neutron oscillation with the probability $P(n \rightarrow \bar{n}) \propto \sin ^{2}((\Delta M / 2) t)$ implies the mass splitting of auxiliary Majorana-type fermions as in (6) and (8). Phenomenologically, one thus observes that the $\gamma^{0}$-parity violation by $\epsilon_{1} \neq 0$ in (5), which gives rise to $M_{+} \neq M_{-}$, is the necessary condition of neutron oscillation. The observation of the neutron oscillation thus implies the dynamical inconsistency of the doublet representation of $\gamma^{0}$ parity. In contrast, the failure (or success) of $i \gamma^{0}$ parity does not tell the presence or absence of neutron oscillation.

In the following, we shall make more precise the role of $\gamma^{0}$ parity and show that the main features of the solutions of the Lagrangian (5) can be obtained from general symmetry considerations. We first write the action corresponding to the Lagrangian (5) in the notation of $n(x)$ and $n^{c}(x)$ as

$$
\begin{aligned}
S\left(n, n^{c}, \epsilon_{1}\right)= & \int d^{4} x\left\{\frac{1}{2} \bar{n}(x)\left[i \gamma^{\mu} \partial_{\mu}-m\right] n(x)\right. \\
& +\frac{1}{2} \overline{n^{c}}(x)\left[i \gamma^{\mu} \partial_{\mu}-m\right] n^{c}(x) \\
& -\frac{i}{2} \epsilon_{1}\left[e^{i \alpha} \overline{n^{c}}(x) n(x)-e^{-i \alpha} \bar{n}(x) n^{c}(x)\right] \\
& \left.-\frac{i}{2} \epsilon_{5}\left[\overline{n^{c}}(x) \gamma_{5} n(x)+\bar{n}(x) \gamma_{5} n^{c}(x)\right]\right\} .
\end{aligned}
$$

The $C P$ (or $T$ ) symmetry of the effective Lagrangian is fixed by the choice of $\alpha$ in (13), and therefore we start with an arbitrary $\alpha$. The quadratic Lagrangian can be diagonalized, and the general solution corresponding to the eigenvalue $M\left(\epsilon_{1}\right)$,

$$
\left[i \gamma^{\mu} \partial_{\mu}-M\left(\epsilon_{1}\right)\right] \psi_{+}\left(x ; \epsilon_{1}\right)=0,
$$

is written as 
PARITY-DOUBLET REPRESENTATION OF MAJORANA ...

$\psi_{+}\left(x ; \epsilon_{1}\right) \equiv c_{1} n(x)+c_{2} \gamma_{5} n(x)+c_{3} n^{c}(x)+c_{4} \gamma_{5} n^{c}(x)$,

with suitable complex constants $\left\{c_{j}\left(\epsilon_{1}\right)\right\}$ that generally depend on $\epsilon_{1}$ (as well as on $m, \epsilon_{5}$, and $\alpha$, but these parameters do not influence the transformation property under the $\gamma^{0}$ parity). One can easily ascertain that the action (13) is invariant under the $\gamma^{0}$-parity transformation of $n(x)$ and $n^{c}(x)$ combined with the inversion $\epsilon_{1} \rightarrow-\epsilon_{1}$, namely,

$$
S\left(n, n^{c}, \epsilon_{1}\right)=S\left(n^{p},\left(n^{c}\right)^{p},-\epsilon_{1}\right) .
$$

Consequently, if one performs a $\gamma_{0}$-parity transformation and the inversion $\epsilon_{1} \rightarrow-\epsilon_{1}$ on the solution (14), one finds a solution of $S\left(n^{p},\left(n^{c}\right)^{p},-\epsilon_{1}\right)$,

$$
\begin{aligned}
\psi_{+}^{p}\left(x ;-\epsilon_{1}\right)= & {\left[c_{1}\left(-\epsilon_{1}\right) n^{p}(x)+c_{2}\left(-\epsilon_{1}\right) \gamma_{5} n^{p}(x)\right.} \\
& \left.+c_{3}\left(-\epsilon_{1}\right)\left(n^{c}(x)\right)^{p}+c_{4}\left(-\epsilon_{1}\right) \gamma_{5}\left(n^{c}(x)\right)^{p}\right] \\
= & \gamma^{0}\left[c_{1}\left(-\epsilon_{1}\right) n(t,-\vec{x})-c_{2}\left(-\epsilon_{1}\right) \gamma_{5} n(t,-\vec{x})\right. \\
& \left.-c_{3}\left(-\epsilon_{1}\right) n^{c}(t,-\vec{x})+c_{4}\left(-\epsilon_{1}\right) \gamma_{5} n^{c}(t,-\vec{x})\right] \\
\equiv & \gamma^{0} \psi_{-}\left(t,-\vec{x} ;-\epsilon_{1}\right),
\end{aligned}
$$

which satisfies (as it will be justified below using the WardTakahashi identity)

$$
\left[i \gamma^{\mu} \partial_{\mu}-M\left(-\epsilon_{1}\right)\right] \psi_{+}^{p}\left(x ;-\epsilon_{1}\right)=0 .
$$

This relation implies

$$
\begin{aligned}
& \gamma^{0}\left[i \gamma^{\mu} \partial_{\mu}-M\left(-\epsilon_{1}\right)\right] \psi_{+}^{p}\left(x ;-\epsilon_{1}\right) \\
& =\gamma^{0}\left[i \gamma^{\mu} \partial_{\mu}-M\left(-\epsilon_{1}\right)\right] \gamma^{0} \psi_{-}\left(t,-\vec{x} ;-\epsilon_{1}\right)=0 .
\end{aligned}
$$

Thus, we have found a solution of $S\left(n^{p},\left(n^{c}\right)^{p},-\epsilon_{1}\right)=$ $S\left(n, n^{c}, \epsilon_{1}\right)$, corresponding to the eigenvalue $M\left(-\epsilon_{1}\right)$ :

$$
\left[i \gamma^{\mu} \partial_{\mu}-M\left(-\epsilon_{1}\right)\right] \psi_{-}\left(x ;-\epsilon_{1}\right)=0 .
$$

The $\gamma^{0}$-parity transformation maps a solution $\psi_{+}\left(x ; \epsilon_{1}\right)$ with mass $M\left(\epsilon_{1}\right)$ in (14) to another solution $\psi_{-}\left(x ;-\epsilon_{1}\right)$ with mass $M\left(-\epsilon_{1}\right)$ in (20) as is suggested by the broken parity symmetry relation in (16).

We return now to the justification of the mass eigenvalue $M\left(-\epsilon_{1}\right)$ in Eq. (18). We note that the solution $\left[i \gamma^{\mu} \partial_{\mu}-\right.$ $\left.M\left(-\epsilon_{1}\right)\right] \psi_{+}^{p}\left(x ;-\epsilon_{1}\right)=0$ of the action $S\left(n^{p},\left(n^{c}\right)^{p},-\epsilon_{1}\right)$ in (18) and the solution $\left[i \gamma^{\mu} \partial_{\mu}-M\left(-\epsilon_{1}\right)\right] \psi_{+}\left(x ;-\epsilon_{1}\right)=0$ of the action $S\left(n, n^{c},-\epsilon_{1}\right)$ share the same mass eigenvalue since one can regard $n \rightarrow n^{p}$ and $n^{c} \rightarrow\left(n^{c}\right)^{p}$ as a formal renaming of field variables. Remark, however, that $S\left(n, n^{c},-\epsilon_{1}\right) \neq S\left(n, n^{c}, \epsilon_{1}\right)$, while $S\left(n^{p},\left(n^{c}\right)^{p},-\epsilon_{1}\right)=$ $S\left(n, n^{c}, \epsilon_{1}\right)$. A justification of (18) is given by a WardTakahashi identity for broken parity symmetry in the path integral by starting with
PHYSICAL REVIEW D 94, 115009 (2016)

$$
\begin{aligned}
& \left.\left\langle T^{\star} n^{p}(x) \overline{\left(n^{c}\right)^{p}}(y)\right\rangle\right|_{\left(\varepsilon_{1}\right)}=\int \mathcal{D} n \mathcal{D} n^{c} n^{p}(x) \overline{\left(n^{c}\right)^{p}}(y) e^{i S\left(n, n^{c}, \epsilon_{1}\right)} \\
& =\int \mathcal{D} n^{p} \mathcal{D}\left(n^{c}\right)^{p} n^{p}(x) \overline{\left(n^{c}\right)^{p}}(y) e^{i S\left(n^{p},\left(n^{c}\right)^{p},-\epsilon_{1}\right)}
\end{aligned}
$$

where $T^{\star}$ stands for time ordering and we used the parity invariance of the path integral measure $\mathcal{D} n^{p} \mathcal{D}\left(n^{c}\right)^{p}=$ $\mathcal{D} n \mathcal{D} n^{c}$ and the broken parity relation (16), $S\left(n^{p}\right.$, $\left.\left(n^{c}\right)^{p},-\epsilon_{1}\right)=S\left(n, n^{c}, \epsilon_{1}\right)$. The last path integral in (21) is identical to the path integral in

$$
\left.\left\langle T^{\star} n(x) \overline{n^{c}}(y)\right\rangle\right|_{\left(-\epsilon_{1}\right)}=\int \mathcal{D} n \mathcal{D} n^{c} n(x) \overline{n^{c}}(y) e^{i S\left(n, n^{c},-\epsilon_{1}\right)},
$$

using the renaming of path integral variables. We thus conclude that $\left.\left\langle T^{\star} n^{p}(x) \overline{\left(n^{c}\right)^{p}}(y)\right\rangle\right|_{\left(\epsilon_{1}\right)}$ defined by $S\left(n, n^{c}, \epsilon_{1}\right)$ agrees with $\left.\left\langle T^{\star} n(x) \overline{n^{c}}(y)\right\rangle\right|_{\left(-\epsilon_{1}\right)}$ defined by $S\left(n, n^{c},-\epsilon_{1}\right)$. This relation holds for other combinations of fields such as $\left\langle T^{\star} n^{p}(x) n^{p}(y)\right\rangle$ also, and thus we conclude that

$$
\left\langle T^{\star} \psi_{+}^{p}\left(x ;-\epsilon_{1}\right) \overline{\psi_{+}^{p}}\left(y ;-\epsilon_{1}\right)\right\rangle
$$

for the action $S\left(n, n^{c}, \epsilon_{1}\right)=S\left(n^{p},\left(n^{c}\right)^{p},-\epsilon_{1}\right)$ agrees with

$$
\left\langle T^{\star} \psi_{+}\left(x ;-\epsilon_{1}\right) \overline{\psi_{+}}\left(y ;-\epsilon_{1}\right)\right\rangle
$$

for the action $S\left(n, n^{c},-\epsilon_{1}\right)$; i.e., they have the same pole mass, $M\left(-\epsilon_{1}\right)$. This provides a justification of the mass eigenvalue in (18).

A similar analysis in the inverse direction starting with the solution $\psi_{-}\left(x ;-\epsilon_{1}\right)$ in (20) of $S\left(n^{p},\left(n^{c}\right)^{p},-\epsilon_{1}\right)$ leads to a solution $\psi_{+}\left(t,-\vec{x} ; \epsilon_{1}\right)$ of $S\left(n,\left(n^{c}\right), \epsilon_{1}\right)$ with mass $M\left(\epsilon_{1}\right)$. We have thus established the $\gamma^{0}$-parity doublet representation $\left\{\psi_{+}\left(x ; \epsilon_{1}\right), \psi_{-}\left(x ;-\epsilon_{1}\right)\right\}$ of the solutions of $S\left(n, n^{c}, \epsilon_{1}\right)$,

$$
\begin{aligned}
& \psi_{+}\left(x ; \epsilon_{1}\right) \stackrel{P}{\rightarrow} \gamma^{0} \psi_{-}\left(t,-\vec{x} ;-\epsilon_{1}\right), \\
& \psi_{-}\left(x ;-\epsilon_{1}\right) \stackrel{P}{\rightarrow} \gamma^{0} \psi_{+}\left(t,-\vec{x} ; \epsilon_{1}\right),
\end{aligned}
$$

which satisfy $\mathrm{P}^{2}=1$ (parity-doublet theorem). This representation is valid irrespective of whether the $\gamma^{0}$ parity is conserved or not. The $\gamma^{0}$-parity violation by $\epsilon_{1} \neq 0$ in (13) is a necessary condition of neutron oscillation, which requires $M_{+}=M\left(\epsilon_{1}\right) \neq M\left(-\epsilon_{1}\right)=M_{-}$, in which case the doublet representation is dynamically inconsistent.

A more detailed specification of the solutions is possible if one assumes some symmetry of the Lagrangian. For example, good $C$ in (5) and (13) with $\alpha=-\pi / 2$ implies the relation $\psi_{+}^{c}=C \bar{\psi}_{+}^{T}$, where the left-hand side $\psi_{+}^{c}$ is evaluated in terms of $n$ and $n^{c}$ by a unitary $C$ transformation, 
$\psi_{+}^{c}\left(x ; \epsilon_{1}\right)=c_{1} n^{c}(x)+c_{2} \gamma_{5} n^{c}(x)+c_{3} n(x)+c_{4} \gamma_{5} n(x)$,

while the right-hand side is evaluated directly from $\psi_{+}$,

$C{\overline{\psi_{+}}}^{T}=i \gamma^{2}\left[c_{1} n(x)+c_{2} \gamma_{5} n(x)+c_{3} n^{c}(x)+c_{4} \gamma_{5} n^{c}(x)\right]^{*}$,

and one obtains

$$
\psi_{+}(x)=c_{1} n+i c_{2} \gamma_{5} n+c_{3} n^{c}+i c_{4} \gamma_{5} n^{c}
$$

as a general expansion analogous to (15) where all the coefficients are now real. The condition $\psi_{+}^{c}=\psi_{+}$and the parity doublet condition $\psi_{+} \rightarrow \gamma^{0} \psi_{-}$in (25) then fix the general forms

$$
\begin{aligned}
& \psi_{+}=\left(c_{1}\left(\epsilon_{1}\right)+i c_{2}\left(\epsilon_{1}\right) \gamma_{5}\right)\left(n+n^{c}\right), \\
& \psi_{-}=\left(c_{1}\left(-\epsilon_{1}\right)-i c_{2}\left(-\epsilon_{1}\right) \gamma_{5}\right)\left(n-n^{c}\right),
\end{aligned}
$$

and the last expression $\psi_{-}$also satisfies the condition $\psi_{-}^{c}=-\psi_{-}$. This is precisely the structure obtained earlier by direct calculations in Eq. (9). Thus, we can derive the general features of the exact solution in (9) without solving explicitly the equations of motion but just by using the $\gamma^{0}$ parity.

The absence of the $\gamma^{0}$ parity-violating $\epsilon_{1}$ term implies the absence of the conventional neutron oscillation because of the mass degeneracy of the two solutions in (25), despite the presence of the $\epsilon_{5}$ term with $\Delta B=2$, which breaks generally defined parity in (13). We discuss what happens in this case by setting $\epsilon_{1}=0$ in (13) which preserves $C$ and $P$. The solution of this Lagrangian with $\epsilon_{1}=0$ is given by (9),

$$
\left(\begin{array}{c}
n(x) \\
n^{c}(x)
\end{array}\right)=\left(\begin{array}{c}
\cos \phi N_{+}(x)-i \gamma_{5} \sin \phi N_{-}(x) \\
\cos \phi N_{-}(x)-i \gamma_{5} \sin \phi N_{+}(x)
\end{array}\right)
$$

but now with

$$
N_{ \pm}(x)=\left[\psi_{+}(x) \pm \psi_{-}(x)\right] / \sqrt{2}
$$

and

$$
\sin 2 \phi \equiv \epsilon_{5} / \sqrt{m^{2}+\epsilon_{5}^{2}},
$$

which satisfy $N_{ \pm}^{c}(x)=N_{\mp}(x)$ and $N_{ \pm}^{p}(x)= \pm \gamma^{0} N_{ \pm}(t,-\vec{x})$ using (11) and (12). The fields $N_{ \pm}(x)$ have the degenerate mass

$$
M=\sqrt{m^{2}+\epsilon_{5}^{2}}
$$

In the notation of (15) and (25), the solution with good $\gamma^{0}$ parity gives a degenerate pair of Majorana fermions or equivalently a Dirac fermion $N_{+}(x)$, and good $C$ implies the doublet representation $\left\{N_{+}(x), N_{+}^{c}(x)\right\}$.

When one generates the neutron, one obtains

$$
n(x)=\cos \phi N_{+}(x)-i \gamma_{5} \sin \phi N_{+}^{c}(x)
$$

in (30) but no oscillation in the conventional sense due to the mass degeneracy, and thus it may appear that there are no physical effects. But $n(x)$ and $n^{c}(x)$ are not orthogonal in the sense

$$
\left\langle T^{\star} n^{c}(x) \bar{n}(y)\right\rangle=\int \frac{d^{4} p}{(2 \pi)^{4}} \frac{\gamma_{5} M \sin 2 \phi}{p^{2}-M^{2}+i \epsilon} e^{-i p(x-y)},
$$

which shows that $n(x)$ decays through $n \rightarrow p+e+\bar{\nu}_{e}$ or $n^{c} \rightarrow \bar{p}+e^{+}+\nu_{e}$, and $n(x)$ annihilates when it collides with ordinary matter containing the neutron. The implication of the absence of oscillation (with $\Delta B=2$ terms present) is the absence of the "bunching effect" in the sense that one would observe predominantly $n^{c}(x)$ starting with $n(x)$ when observed at a proper moment in the presence of oscillation.

In analogy of the neutron oscillation to BCS theory [11], we note that the relation (30) is precisely a Lorentzinvariant version of the Bogoliubov transformation [13] which diagonalizes the Lagrangian (with $\epsilon_{1}=0$ ) by preserving the anticommutation relations

$$
\left\{n(t, \vec{x}), n^{c}(t, \vec{y})\right\}=\left\{N_{+}(t, \vec{x}), N_{+}^{c}(t, \vec{y})\right\}
$$

and $\gamma^{0}$ parity. An interesting aspect of the relativistic Bogoliubov transformation is that (35) implies

$$
\left\{\dot{n}^{c}(t, \vec{x}), \bar{n}(t, \vec{y})\right\} \neq 0,
$$

if one applies the Bjorken-Johnson-Low (BJL) prescription, and thus $n^{c}(x)$ is dynamically correlated with $\bar{n}(x)$ although $\left\{n^{c}(t, \vec{x}), \bar{n}(t, \vec{y})\right\}=0$. More interestingly, our parity-doublet theorem implies that the mass splitting of Majorana-type quasifermions may appear also in condensed matter physics, if parity ( $\gamma^{0}$ parity) is violated, although our analysis is strictly valid for the quadratic (mean field) approximation.

\section{IV. $C P$ AND RELATED ISSUES}

It is well known that Majorana neutrinos modify the $C P$ property in electroweak interactions [14]. For example, one can in principle have $C P$ violation in the model with only two generations, although such extra $C P$ violation is not observable in neutrino oscillation [14]. We discuss the $C P$ properties in the neutron oscillation using the explicit Lagrangian (13), which is $C P$ violating for $\alpha \neq 0$. 
To solve (13), we first use the Bogoliubov transformation (30) as a change of variables, which preserves $C$ and $P$. Setting $N_{+}=N$, the Lagrangian (13) is then written as

$$
\begin{aligned}
\mathcal{L}= & (1 / 2) \bar{N}\left[i \not \partial-M-i \epsilon_{1} \gamma_{5} \sin \alpha \sin 2 \phi\right] N \\
& +(1 / 2) \overline{N^{c}}\left[i \not \partial-M-i \epsilon_{1} \gamma_{5} \sin \alpha \sin 2 \phi\right] N^{c} \\
& -(i / 2) \epsilon_{1} e^{i \tilde{\alpha}} \sqrt{1-(\sin \alpha \sin 2 \phi)^{2}} \overline{N^{c}} N+\text { H.c. },
\end{aligned}
$$

where $\quad \sin \tilde{\alpha}=\sin \alpha \cos 2 \phi / \sqrt{1-(\sin \alpha \sin 2 \phi)^{2}}$. After performing $N \rightarrow e^{-i \tilde{\alpha} / 2} \tilde{N}, C P$ violation appears only in the parity-violating mass term. A further transformation

$$
\frac{1}{\sqrt{2}}\left(\begin{array}{c}
\tilde{N}(x)-i \tilde{N}^{c}(x) \\
\tilde{N}(x)+i \tilde{N}^{c}(x)
\end{array}\right)=\left(\begin{array}{c}
\tilde{\varphi}_{+}(x) \\
\tilde{\varphi}_{-}(x)
\end{array}\right)
$$

leads to

$$
\begin{aligned}
\mathcal{L}= & (1 / 2) \overline{\tilde{\varphi}}_{+}\left[i \not \partial-\left(M+\epsilon_{1} \sqrt{1-\left(\tilde{\epsilon}_{1} / \epsilon_{1}\right)^{2}}\right)-i \tilde{\epsilon}_{1} \gamma_{5}\right] \tilde{\varphi}_{+} \\
& +(1 / 2) \tilde{\tilde{\varphi}}_{-}\left[i \not \partial-\left(M-\epsilon_{1} \sqrt{1-\left(\tilde{\epsilon}_{1} / \epsilon_{1}\right)^{2}}\right)-i \tilde{\epsilon}_{1} \gamma_{5}\right] \tilde{\varphi}_{-},
\end{aligned}
$$

with $\tilde{\epsilon}_{1} \equiv \epsilon_{1} \sin \alpha \sin 2 \phi$. After a suitable chiral transformation, one obtains a pair of Majorana fermions,

$$
\begin{aligned}
\mathcal{L}= & (1 / 2) \bar{\varphi}_{+}(x)\left[i \not \partial-M_{+}\right] \varphi_{+}(x) \\
& +(1 / 2) \bar{\varphi}_{-}(x)\left[i \not \partial-M_{-}\right] \varphi_{-}(x),
\end{aligned}
$$

where $\varphi_{ \pm}=e^{i \theta_{ \pm} \gamma_{5}} \tilde{\varphi}_{ \pm}$with

$$
\left(M \pm \epsilon_{1} \sqrt{1-\left(\tilde{\epsilon}_{1} / \epsilon_{1}\right)^{2}}\right)+i \tilde{\epsilon}_{1} \gamma_{5} \equiv M_{ \pm} e^{2 i \theta_{ \pm} \gamma_{5}},
$$

and

$$
M_{ \pm}=\left(\left[M \pm \epsilon_{1} \sqrt{1-\left(\tilde{\epsilon}_{1} / \epsilon_{1}\right)^{2}}\right]^{2}+\left(\tilde{\epsilon}_{1}\right)^{2}\right)^{1 / 2} .
$$

This mass formula covers the special cases (6) and (8) by choosing $\alpha=0$ and $\alpha=-\pi / 2$, respectively.

To analyze the possible effects of $C P$ violation, we first look at the parity-violating mass term for the Dirac fermion in (38), which is written

$$
\mathcal{L}_{\text {mass }} \simeq-\left(\epsilon_{1} \epsilon_{5} / M\right) \sin \alpha \bar{n}(x) i \gamma_{5} n(x),
$$

in the leading order in $\epsilon_{1}$ and $\epsilon_{5}$. This term, which is used to evaluate the neutron electric dipole moment, should be added to the contributions from other sources such as the QCD $\theta$ vacuum.

To examine the more direct effects of $C P$ violation in the oscillation amplitude, we write the exact solution of the neutron field using (30), (39), and (40),

$$
\begin{aligned}
n(x)= & (1 / \sqrt{2})\left[\cos \phi e^{-i \tilde{\alpha} / 2}+\gamma_{5} \sin \phi e^{i \tilde{\alpha} / 2}\right] e^{-i \theta_{+} \gamma_{5}} \varphi_{+}(x) \\
& +(1 / \sqrt{2})\left[\cos \phi e^{-i \tilde{\alpha} / 2}-\gamma_{5} \sin \phi e^{i \tilde{\alpha} / 2}\right] e^{-i \theta_{-} \gamma_{5}} \varphi_{-}(x), \\
n^{c}(x)= & (-i / \sqrt{2})\left[\cos \phi e^{i \tilde{\alpha} / 2}-\gamma_{5} \sin \phi e^{-i \tilde{\alpha} / 2}\right] e^{-i \theta_{-} \gamma_{5}} \varphi_{+}(x) \\
& +(i / \sqrt{2})\left[\cos \phi e^{i \tilde{\alpha} / 2}+\gamma_{5} \sin \phi e^{-i \tilde{\alpha} / 2}\right] e^{-i \theta_{+} \gamma_{5}} \varphi_{-}(x) .
\end{aligned}
$$

We choose the $P$ and $C$ transformation laws of the mass eigenstates (40) consistent with the doublet representation of $\gamma^{0}$ parity as in (25) [12],

$$
\varphi_{ \pm}^{p}(x)=\gamma^{0} \varphi_{\mp}(t,-\vec{x}), \quad \varphi_{ \pm}^{c}(x)= \pm i \varphi_{\mp}(x),
$$

which induce $n \rightarrow \gamma^{0} n$ and $n^{c} \rightarrow-\gamma^{0} n^{c}$, and $n \rightarrow n^{c}$ in (44), respectively, for the vanishing $C P$-breaking parameter $\alpha=0$. The $C P$ symmetry expressed by

$$
\varphi_{ \pm}^{c p}(x)= \pm i \gamma^{0} \varphi_{ \pm}(t,-\vec{x})
$$

does not induce the $C P$ transformation of $n$ and $n^{c}$ for $\alpha \neq 0$, which shows the $C P$ breaking in (44); to be precise, the $C P$ transformation of $\varphi_{ \pm}(x)$ in the expression of $n(x)$ does not lead to $-\gamma^{0} n^{c}(t,-\vec{x})$. We regard (44) as an analog of the "mixing matrix" between the flavor eigenstates $\left(n, n^{c}\right)$ and mass eigenstates $\left(\varphi_{+}, \varphi_{-}\right)$, and we test the above $C P$ breaking in the neutron oscillation.

One can then evaluate the oscillation probability amplitude $A=A\left(n(\vec{p}) \rightarrow n^{c}(\vec{p}) ; t\right)$ by

$$
A=\left\langle n_{R}^{c}(\vec{p}) ; 0 \mid n_{R}(\vec{p}) ; t\right\rangle+\left\langle n_{L}^{c}(\vec{p}) ; 0 \mid n_{L}(\vec{p}) ; t\right\rangle,
$$

where we used the chirally projected states to take care of the $\gamma^{5}$ appearing in the above solution. Thus,

$$
\begin{aligned}
A= & \frac{i}{2}\left[\cos ^{2} \phi e^{-i \tilde{\alpha}}-\sin ^{2} \phi e^{i \tilde{\alpha}}\right] \\
& \times\left\{e^{-i \theta-i E_{+} t}\left\langle\varphi_{R,+}(\vec{p}, 0) \mid \varphi_{R,+}(\vec{p}, 0)\right\rangle\right. \\
& -e^{i \theta-i E_{-} t}\left\langle\varphi_{R,-}(\vec{p}, 0) \mid \varphi_{R,-}(\vec{p}, 0)\right\rangle \\
& +e^{i \theta-i E_{+} t}\left\langle\varphi_{L,+}(\vec{p}, 0) \mid \varphi_{L,+}(\vec{p}, 0)\right\rangle \\
& \left.-e^{-i \theta-i E_{-} t}\left\langle\varphi_{L,-}(\vec{p}, 0) \mid \varphi_{L,-}(\vec{p}, 0)\right\rangle\right\},
\end{aligned}
$$

where we defined $\theta=\theta_{+}-\theta_{-}$. We now note

$$
\begin{aligned}
\left\langle\varphi_{R,+}(\vec{p}, 0) \mid \varphi_{R,+}(\vec{p}, 0)\right\rangle & =\left\langle\varphi_{L,+}(\vec{p}, 0) \mid \varphi_{L,+}(\vec{p}, 0)\right\rangle \\
& =\frac{1}{2}\left\langle\varphi_{+}(\vec{p}, 0) \mid \varphi_{+}(\vec{p}, 0)\right\rangle
\end{aligned}
$$

and similarly for $\varphi_{-}(\vec{p}, 0)$ using the $i \gamma^{0}$-parity invariance of free Majorana equations, namely, $\varphi_{R,+}^{p}(x)=i \gamma^{0} \varphi_{L,+}(t,-\vec{x})$. Using the normalization of states $\left\langle\varphi_{+}(\vec{p}, 0) \mid \varphi_{+}(\vec{p}, 0)\right\rangle=$ $\left\langle\varphi_{-}(\vec{p}, 0) \mid \varphi_{-}(\vec{p}, 0)\right\rangle$, we find the amplitude 


$$
\begin{aligned}
A= & {\left[\cos ^{2} \phi e^{-i \tilde{\alpha}}-\sin ^{2} \phi e^{i \tilde{\alpha}}\right] \cos \theta \sin \left(\frac{1}{2} \Delta E t\right) } \\
& \times e^{-i \bar{E} t}\left\langle\varphi_{+}(\vec{p}, 0) \mid \varphi_{+}(\vec{p}, 0)\right\rangle,
\end{aligned}
$$

with $\Delta E=E_{+}-E_{-}$and $\bar{E}=\left(E_{+}+E_{-}\right) / 2$. Adopting $\left|\left\langle\varphi_{+}(\vec{p}, 0) \mid \varphi_{+}(\vec{p}, 0)\right\rangle\right|^{2}=1$, one obtains the oscillation probability,

$$
P\left(n(\vec{p}) \rightarrow n^{c}(\vec{p}) ; t\right)=\left(1-\sin ^{2} 2 \phi \cos ^{2} \tilde{\alpha}\right) \cos ^{2} \theta \sin ^{2}\left(\frac{1}{2} \Delta E t\right) .
$$

By recalling the definitions of $\sin \tilde{\alpha}$ in (38) and $\theta_{ \pm}$and $M_{ \pm}$in (41), the $C P$ transformation, which is equivalent to $\alpha \rightarrow-\alpha$, corresponds to

$$
\tilde{\alpha} \rightarrow-\tilde{\alpha}, \quad \theta=\theta_{+}-\theta_{-} \rightarrow-\theta,
$$

and the above oscillation probability (51) and the energy difference $\Delta E=\sqrt{\vec{p}^{2}+M_{+}^{2}}-\sqrt{\vec{p}^{2}+M_{-}^{2}}$ are all invariant. Although $\alpha$ modifies the magnitudes of $\Delta E$ (and thus the oscillation time) and probability $P$ themselves, we do not regard these modifications as a manifestation of $C P$ violation in oscillation which is typically expressed by $P\left(n(\vec{p}) \rightarrow n^{c}(\vec{p}) ; t\right) \neq P\left(n^{c}(\vec{p}) \rightarrow n(\vec{p}) ; t\right)$. We observe no direct $C P$ violation in the neutron oscillation in vacuum.

Note that we discussed $C P$ by looking at only the neutron sector assuming that the flavor degree $[(p, n)$ multiplet structure, for example] is fixed by the baryon number-conserving sector of the full model, unlike the neutrino oscillation where a combination of neutrino and charged-lepton mixing matrices is analyzed. See also Refs. [7-9] for related analyses.

The possible $C P T$ violation in the hadron sector appears to be very small as is indicated by both experimental limit $\mid m_{K}-$ $m_{\bar{K}} \mid<0.44 \times 10^{-18} \mathrm{GeV}$ [15] and a recent model study within an extension of the Standard Model [16]. However, we mention that the relevant mass scale of the neutron oscillation is estimated at $\epsilon_{1} \leq 6 \times 10^{-24} \mathrm{eV}$ and $n-\bar{n}$ mass splitting itself is constrained to be less than $10^{-15} \mathrm{eV}$ to observe the oscillation [6]. These values are not much different from the estimated mass difference $\sim 10^{-20} \mathrm{eV}$ of the electron and positron induced by the possible $C P T$ breaking that is required to explain the small $(2 \sigma)$ mass difference of the observed Sun neutrino and reactor antineutrino as really arising from the Lorentz-invariant $C P T$ breaking [16]. See also a recent paper [10].

In conclusion, we have clarified the full physical contents of the model of neutron oscillation (5). It has been shown that basic notions such as the parity doubling of Majorana fermions and the Bogoliubov transformation play main roles in the analysis of the model (5), which, as a relativistic analog of BCS theory, is in turn suggestive of new possibilities in condensed matter physics.

\section{ACKNOWLEDGMENTS}

We thank Masud Chaichian for very helpful discussions. This work is supported in part by the Vilho, Yrjö, and Kalle Väisälä Foundation. The support of the Academy of Finland under Projects No. 136539 and No. 272919 is gratefully acknowledged.
[1] S. Bilenky, Introduction to the Physics of Massive and Mixed Neutrinos (Springer, Berlin, 2010), Vol. 817.

[2] A. Y. Kitaev, AIP Conf. Proc. 1134, 22 (2009); S. Ryu, A. P. Schnyder, A. Furusaki, and A. W. W. Ludwig, New J. Phys. 12, 065010 (2010).

[3] J. D. Bjorken and S. D. Drell, Relativistic Quantum Fields (McGraw-Hill, New York, 1965).

[4] S. Weinberg, The Quantum Theory of Fields I (Cambridge University Press, Cambridge, England, 1995).

[5] R. N. Mohapatra and R. E. Marshak, Phys. Rev. Lett. 44, 1316 (1980); see also V. A. Kuzmin, JETP Lett. 12, 228 (1970); S. L. Glashow, Proceedings of 1979 Cargèse Summer Institute on Quarks and Leptons, edited by M. Lévy et al. (Plenum, New York, 1980), p. 687; L. N. Chang and N. P. Chang, Phys. Lett. 92B, 103 (1980); R. N. Mohapatra and R.E. Marshak, Phys. Lett. 94B, 183 (1980); T. K. Kuo and S. Love, Phys. Rev. Lett. 45, 93 (1980); K. G. Chetyrkin, M. V. Kazarnovsky, V. A. Kuzmin, and M. E. Shaposhnikov, Phys. Lett. 99B, 358 (1981); S. Rao and R. E. Shrock, Phys. Lett. B 116, 238 (1982).
[6] R. N. Mohapatra, J. Phys. G 36, 104006 (2009); D. G. Phillips, II et al., Phys. Rep. 612, 1 (2016).

[7] Z. Berezhiani and A. Vainshtein, arXiv:1506.05096v2.

[8] K. Fujikawa and A. Tureanu, arXiv:1510.00868.

[9] D. McKeen and A. E. Nelson, Phys. Rev. D 94, 076002 (2016).

[10] S. Gardner and X. Yan, Phys. Rev. D 93, 096008 (2016).

[11] L. N. Chang and N. P. Chang, Phys. Rev. Lett. 45, 1540 (1980).

[12] We assume that the free Majorana particles in Minkowski space respect the full Lorentz symmetry, including $P$ and $T$ and thus $C$, due to $C P T$.

[13] K. Fujikawa and A. Tureanu, arXiv:1609.03309.

[14] S. M. Bilenky, J. Hosek, and S. T. Petcov, Phys. Lett. 94B, 495 (1980); M. Doi, T. Kotani, H. Nishiura, K. Okuda, and E. Takasugi, Phys. Lett. 102B, 323 (1981).

[15] K. A. Olive et al. (Particle Data Group Collaboration), Chin. Phys. C 38, 090001 (2014).

[16] K. Fujikawa and A. Tureanu, Phys. Lett. B 743, 39 (2015); Mod. Phys. Lett. A 30, 1530016 (2015). 